\title{
Design and Development of Long Range Surface Plasmon Resonance Sensor Chip and System for High Sensitivity Detection
}

\author{
Lu-lu ZHANG ${ }^{a}{ }^{*}$, Xing CHEN, Cun-fang XU, Yan-fei REN, Ya-ting LI \\ and Da-fu CUI
}

State Key Laboratory of Transducer Technology, Institute of Electronics, Chinese Academy of Sciences, Beijing, China

allzhang@mail.ie.ac.cn

${ }^{*}$ Corresponding author

Keywords: Surface plasmon resonance (SPR), Long range, Sensitivity, Biosensor.

\begin{abstract}
Long range surface plasmon resonance (LRSPR) was used to improve the propagation lengths, electric field strengths, and the sharpness of angular resonance curves compared with the conventional surface plasmon resonance (CSPR). LRSPR sensor chip was designed with buffer layer whose refractive indices in the vicinity of water. The resonance curves of the LRSPR and conventional SPR were compared under different parameters of multilayer structure by Winspall software. The theoretical calculation showed that the sensitivity of LRSPR method was higher than the conventional SPR. Homemade LRSPR sensor system was constructed with a red laser light source, a P-polarizer, a triangle glass prism, a long range sensor chip and a linear CCD detector. The performance of the LRSPR sensor were tested and compared. The results showed that the sensitivity of LRSPR chip was improved compared with the conventional SPR which was in accordance with the theoretical calculation. LRSPR showed a higher sensitivity and detection range so that it could be used as an excellent living cell sensing method for the real-time monitoring.
\end{abstract}

\section{Introduction}

Surface plasmon resonance (SPR) biosensors were used for direct, label-free detection of chemicals and biological species [1]. It was an extremely sensitive optical technique to detect the changes in refractive index occurring at the sensor interface. Considerable research has been carried out on surface plasmon resonance (SPR) that has a broad range of applications including studies of biomolecular interactions [2], including DNA/DNA, DNA-binding protein, antibody-antigen systems [3-5].

However, for detection of biological agents in low concentrations or cells, the biosensors must provide high-resolution to resolve the changes in refractive index. The refractive index changes were too low to be measured directly and the penetration depth of the evanescent field was not enough to detect cells in conventional surface plasmon resonance (CSPR) biosensors. In order to improve the sensitivity and the penetration depth of the evanescent field for the molecular or cells detection, Long range surface plasmon resonance (LRSPR) biosensors were developed [6].

LRSPR biosensors were special surface plasmon modes which propagated along a thin metal film suspended between two dielectrics with similar refractive indices [7]. Long range surface plasmon resonance (LRSPR) was used to improve the penetration depth, electric field strengths, and the sharpness of angular resonance curves compared 
with the CSPR. Matsubara et al. presented experimental verification of an angle modulated type of an LRSPR-based sensor in 1990[8]. Recently, LRSPR were introduced to Observe biomolecular binding events at metallic surfaces. Charette et al. used LRSPR sensors to detection and monitoring of toxicity with living cells which showed a higher sensitivity for toxicity measurements based on living cells [9]. Thierry et al. systematic studied the SPR signals generated by cells for sensors with different characteristic lengths [10].

In this paper, LRSPR sensors were designed and constructed, especially the procedure of fabrication of the LRSPR sensor chips. The resonance curves of the LRSPR and conventional SPR were compared by calculation and experiment. LRSPR showed a higher sensitivity and detection range.

\section{Experimental}

\section{Sensor Chip}

The CSPR biosensor chip was monolayer metal membrane structure, while the chip of LRSPR biosensor was a sandwich structure which contains buffer layer, metal layer and analyte layer, shown in Figure 1. The thickness of each layer was the main parameter that determines the SPR absorption peak, which directly affected the sensitivity of the sensor. The buffer layer of the LRSPR chip was magnesium fluoride material to obtain a similar refractive index with the analyte.

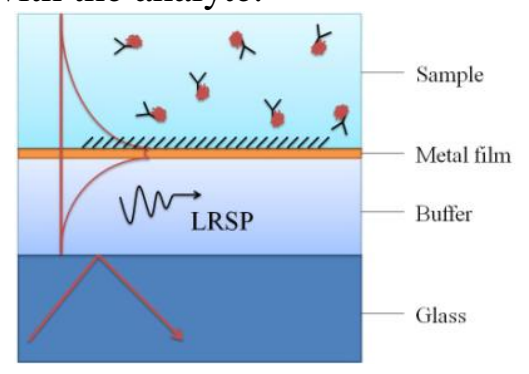

Figure 1. Sandwich structure of the LRSPR sensor chip

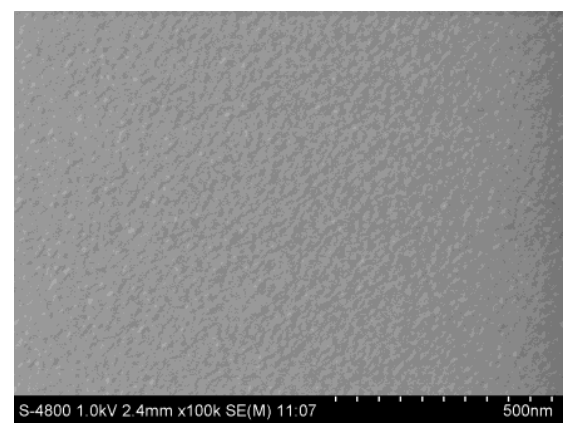

(a)

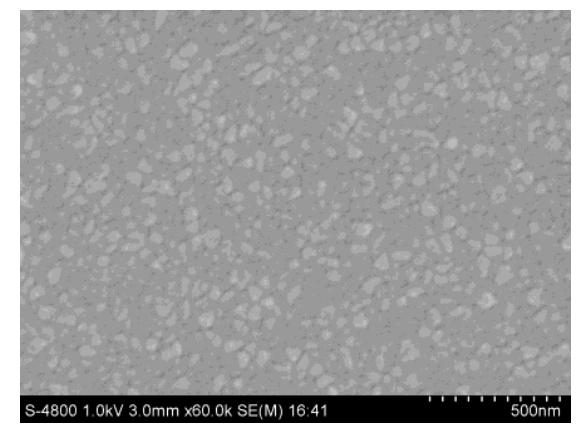

(b)

Figure 2. (a) SEM image of 800nm thick magnesium fluoride evaporated on glass substrates. (b) SEM image of 30nm thick gold film deposited onto the magnesium fluoride layer

The CSPR sensor chips were coated with $2 \mathrm{~nm}$ of chromium then $50 \mathrm{~nm}$ of gold film by magnetron sputtering technology on $20 \times 20 \times 0.2 \mathrm{~mm}^{3}$ BK7 glass substrates. LRSPR sensors evaporated $700 \mathrm{~nm}$ or $800 \mathrm{~nm}$ magnesium fluoride on $20 \times 20 \times 0.2 \mathrm{~mm}^{3} \mathrm{BK} 7$ glass substrates. The SEM image of magnesium fluoride was shown in Figure 2a. The magnesium fluoride islands were observed which showed a good of homogeneity. Then 
30nm gold or platinum films were deposited onto the magnesium fluoride layer by magnetron sputtering technology. The SEM image of the LRSPR chip with 800nm magnesium fluoride and 30nm gold was shown in Figure 2b. As illustrated in Figure 2 the formation of gold islands was bigger than the magnesium fluoride islands.

\section{Flow Cell}

One channel PDMS flow cell immobilized on top of the LRSPR chips was used to imported liquid to the sensor surface for detecting reactions. The flow cell contained a micro flow channel made by a mixture of the PDMS oligomer and the crosslinking agent (Sylgard 184) with a 10:1 ratio. The mixture was degassed under vacuum, poured into a silanized glass mould, and then cured in an oven at $80^{\circ} \mathrm{C}$ for 8 hours. After the glass mould was peeled off, the PDMS flow cell with micro reaction channels were formed. The dimension of the channel was typically $1 \mathrm{~mm}$ in width, and $2 \mathrm{~mm}$ in height.

\section{LRSPR Sensor}

The homemade LRSPR sensor was based on the prism coupling mode of the Kretschmann structure. The coupling angle was sensitive to changes in the refractive index of the medium adjacent to the metal layer supporting surface plasmons. As shown in Figure 3, the proposed LRSPR sensor consisted a red laser light source $(650 \mathrm{~nm}$ peak wavelength), a prism with an equilateral triangle shape ( $\mathrm{n}=1.72$; Beijing Glass Factory, China), LRSPR sensor chip, and a CCD camera (Basler, A102f). The laser, the polarizing filter and the lens system were installed on a rotating arm. The CCD was mounted on another rotating arm and both rotating arms were controlled by a stepper motor. The laser was driven by a home designed light driver module which insured the output powers do not change. All the modules were controlled by the computer through the communication module.

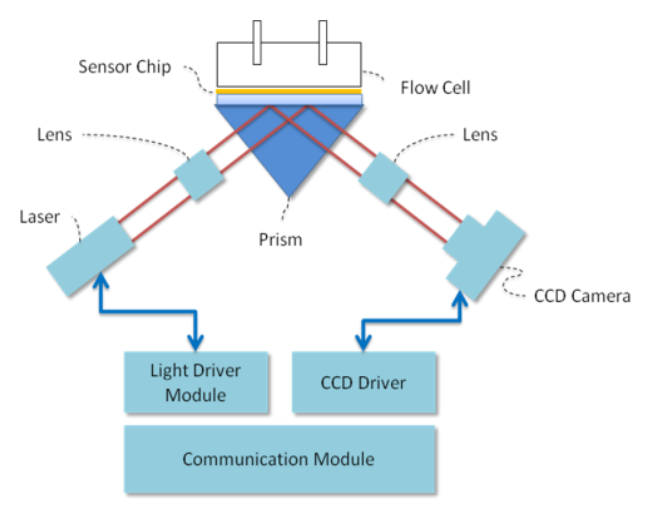

Figure 3. Structure of LRSPR sensor

\section{Results and Discussion}

\section{Theory}

Long range SPR sensor detection was quantitative measurement of reflectivity and resonance angle. Based on the Fresnel formula and the theory of multilayer reflection, the reflection coefficient of the prism/dielectric/metal/dielectric multilayer structure was theoretically analyzed. By means of the equivalent of two adjacent interfaces of the top layer of the multilayer film as an interface, and then through the middle layer, the 
reflection coefficient and reflectivity of the multilayer film have been calculated by the method of calculating the bottom layer.

The multi-layer dielectric constants and the thickness of the films were substituted into the Winspall software to optimize the film thickness ratio and simulate the LRSPR reflectivity curve. CSPR and LRSPR absorption peak curves were compared, as shown in Figure 4. The theoretical analysis showed that the half peak width of the LRSPR was much smaller than that of the CSPR with regard to the same absorption peak depth. The theoretical analysis indicated that the sensitivity of LRSPR method was higher than the CSPR.

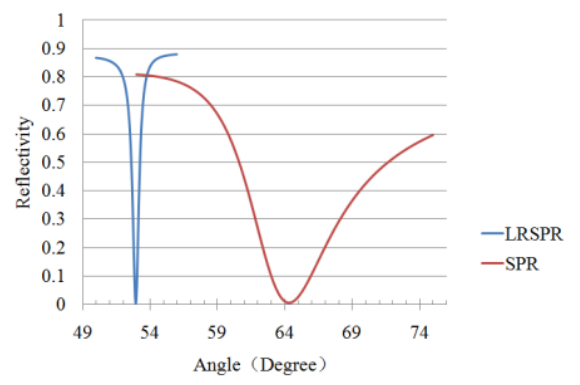

Figure 4. Simulation of CSPR and LRSPR absorption peak curves

In order to optimize the detection sensitivity of LRSPR sensors, the buffer layer thickness was assumed to $700 \mathrm{~nm}$, and $20 \mathrm{~nm}, 30 \mathrm{~nm}$, 40nm thick gold film reflectivity curves were simulated by software shown in Figure 5. It was found that the 30nm thick gold film layer showed a deeper absorption peak curve.

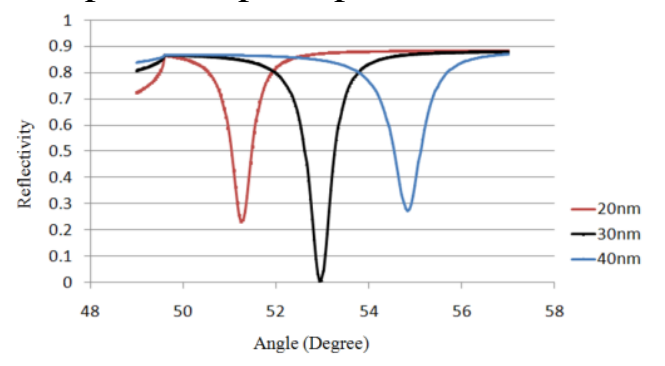

Figure 5. Calculation of the reflectivity curves of LRSPR sensors with $700 \mathrm{~nm}$ thick buffer layer and different thickness of gold film layers.

If the gold film layer was fixed as $30 \mathrm{~nm}$, the thickness of buffer layer was changed as $500 \mathrm{~nm}, 600 \mathrm{~nm}, 700 \mathrm{~nm}, 800 \mathrm{~nm}, 900 \mathrm{~nm}$ separately. The reflectivity curves of LRSPR were calculated, shown in Figure 6. To get higher sensitivity in the LRSPR sensors, thickness parameters were compared under different conditions. Finally narrower half peak width and deeper absorption peak depth was obtained with a parameter of 700nm buffer layer and 30nm gold film layer.

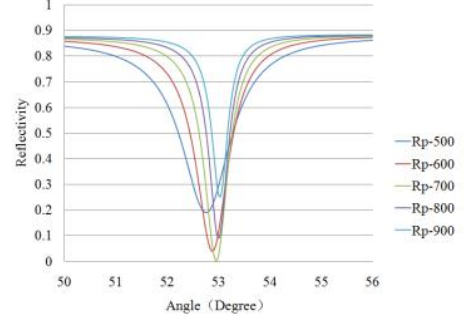

Figure 6. Calculation of the reflectivity curves of LRSPR sensors with 30nm thick gold film layer and different thickness of buffer layers. 


\section{Characteristics of the LRSPR}

For the excitation of LRSPR, 30nm thick gold films or platinum films deposited onto the magnesium fluoride buffer layer. For the excitation of CSPR, 50nm thick gold films or 30nm thick platinum films were deposited directly onto the BK7 glass substrates. The performance of the sensor chips were tested and compared. Figure 7 showed the typical SPR absorption peaks with different thickness of buffer layers and gold film layers. Comparing with the CSPR chip, the resonant dip of LRSPR chip was shifted toward higher steps of the mechanical motor corresponding to the lower angles of incident light and its width was decreased. The reflectivity curves for $700 \mathrm{~nm}$ and 800nm buffer layers were compared whose resonances angles were located at similar angles in accordance with the theoretical analysis.

The similar conclusion could be conducted through the test of platinum film LRSPR sensor chips. The experiment results were shown in Figure 8. Comparing with the CSPR chip, the resonance curve improvement of the platinum film LRSPR chips was more obvious than the gold film LRSPR chip. However, the long-term stability of platinum was poor in many environments, so that improved sensor performance cannot be maintained.

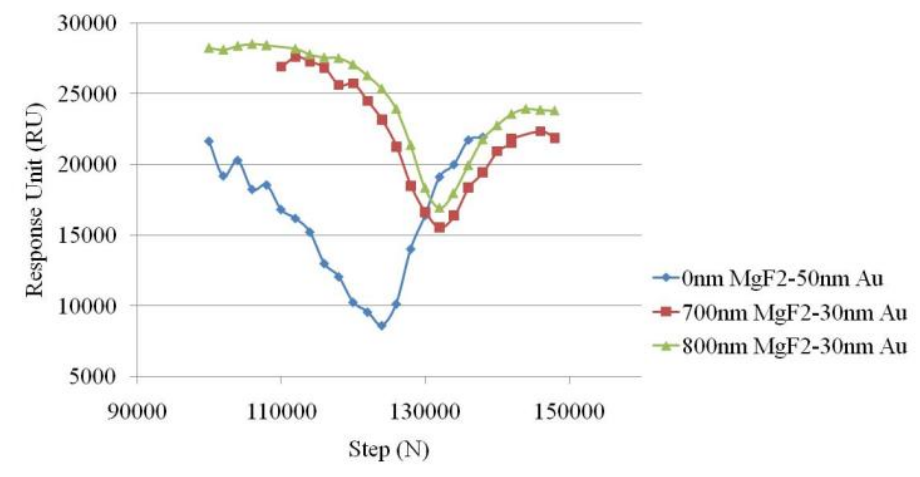

Figure 7. SPR absorption peaks with different thickness of buffer layers and gold film layers.

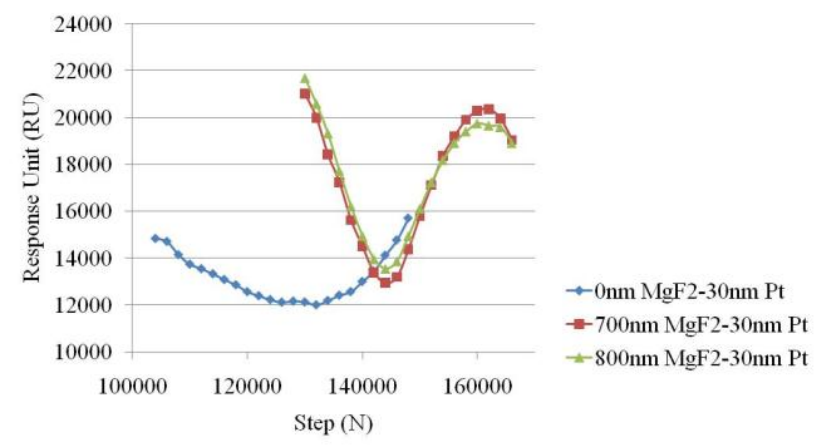

Figure 8. SPR absorption peaks with different thickness of buffer layers and platinum film layers

\section{Conclusions}

LRSPR technology was an effective method to decrease the resonance width and increase the sensitivity of the biosensors. The performance of different the sensor chips were tested and compared. The results showed that the sensitivity of LRSPR chip was improved compared with the conventional SPR. Further optimization of materials and film thickness should to be conducted to achieve higher sensitivities. 


\section{Acknowledgements}

The authors greatly acknowledge the financial support from the National Basic Research Program of China (973 Program) (Grant No. 2014CB744600) and the National Natural Science Foundation of China (Grant Nos. 61571420, 31571007 and 81371711).

\section{References}

[1] J. Homola, Surface plasmon resonance sensors for detection of chemical and biological species, Chemical Reviews, 108 (2008) 462-493.

[2] M. Piliarik, L. Parova, J. Homola, High-throughput SPR sensor for food safety, Biosens. Bioelectron., 24 (2009) 1399-1404.

[3] E.J. Jeong, Y.S. Jeong, K. Park, S.Y.Yi, J. Ahn, S.J. Chung, M. Kim, B.H. Chung, Directed immobilization of DNA-binding proteins on a cognate DNA-modified chip surface, Journal of Biotechnology, 135 (2008) 16-21.

[4] H. Li, H.Y. Cai, X. Chen, J.H. Sun, L.L. Zhang, D.F.Cui, Continuous immunoassay for sulfamethazine by surface Plasmon resonance based biosensor, Analytical Letters, 43 (2010) 499-507.

[5] H. Wu, H. Li, F.Z.H. Chua, S.F.Y. Li, Rapid detection of melamine based on immunoassay using portable surface plasmon resonance biosensor, Sens. Actuators B, 178 (2013) 541-546.

[6] R. Slavík, J. Homola, Ultrahigh resolution long range surface plasmon-based sensor, Sensors \& Actuators B, 123 (2007) 10-12.

[7] D. Sarid, Long-range surface-plasma waves on very thin metal films, Physical Review Letters, 47 (1981) 1927-1930.

[8] K. Matsubara, S. Kawata, S. Minami, Multilayer system for a highprecision surface plasmon resonance sensor, Optics Lett., 15 (1990) 75-77.

[9]V. Chabot, Y. Miron, M. Grandbois, P.G. Charette, Long range surface plasmon resonance for increased sensitivity in living cell biosensing through greater probing depth, Sensors and Actuators B, 174 (2012) 94-101.

[10] R. Méjard, B. Thierry, Systematic study of the surface plasmon resonance signals generated by cells for sensors with different characteristic lengths, Plos One, 9 (2014) e107978. 